\title{
Psychiatric features of new variant Creutzfeldt-Jakob disease
}

\author{
R. G. Will, G. Stewart, M. Zeidler, M. A. Macleod and R. S. G. Knight
}

\begin{abstract}
Alms and method New variant Creutzfeldt-Jakob disease is a novel prion disease of humans that may be causally linked to bovine spongiform encephalopathy. Psychiatric symptoms occur in the early stages of the illness and may be difficult to distinguish from the symptoms of more common psychiatric disorders. Cases of new variant Creutzfeldt-Jakob disease are identified through the national surveillance system. Information on psychiatric features has been obtained by review of case notes and, in the majority of cases, by interview of relatives by a member of the surveillance staff.

Results Thirty-flve cases of new variant CreutzfeldtJakob disease have been identified in the UK and detailed information on the clinical features and investigations is currently available in 33 of these cases. All but one of the cases exhibited prominent early psychiatric symptomatology, but the diagnosis of an underlying neurological disease was not possible in the majority of cases until the development of neurological symptoms and signs. Early indications of an underlying neurological disorder included cognitive impairment, persistent sensory symptoms or limb pain and, in a minority, gait imbalance, dysarthria or visual symptoms. Limited evidence suggests that investigations such as electroencephalogram or brain imaging are unlikely to provde useful diagnostic information during the 'psychiatric' phase of the illness. Clinical implications the early recognition of an underlying neurological disorder may be impossible in the early psychiatric phase of new variant CreutzfeldtJakob disease, but the suspicion of this diagnosis may be raised by the occurrence of associated neurological symptoms. The development of early diagnostic markers is an important objective.
\end{abstract}

New variant Creutzfeldt-Jakob disease (CJD) is a fatal neurological disorder, which is thought to be caused by the agent of bovine spongiform encephalopathy (Will et al, 1996; Bruce et al, 1997). The clinical phenotype of new variant CJD is relatively distinct from sporadic CJD, with an extended illness duration, a younger age at death and predominantly psychiatric symptomatology in the early stages. There have been 35 deaths from new variant $C J D$ in the UK up to 31 December 1998 and the majority have seen a psychiatrist, often as the first specialist referral.
The non-specific characteristics of the psychiatric symptoms in the majority of cases of new variant CJD make early recognition of an underlying neurological disorder difficult or impossible, but it is perhaps timely to review the psychiatric features of this disease and indicate features that may help in diagnosis.

\section{Background}

Thirty-five cases of new variant CJD have been identified in the UK with a mean age at death of 30 years (range 18-53) and a median illness duration of 14 months (range 8-38). Further analysis in this review includes 33 cases, as in two cases there was insufficient detail available for analysis. Psychiatrists were involved in the management of 25 of the 33 cases. In 17 cases a psychiatrist was the first specialist involved. while in eight cases psychiatrists were involved after referral to a neurologist. The proportion of cases seeing a psychiatrist may have changed following the publication of the neurological and psychiatric features of the first 14 cases of new variant CJD (Zeidler et al, 1997a,b). Only one of first 14 cases was not seen by a psychiatrist in comparison to seven of the subsequent 19 cases not seen by a psychiatrist.

The time from clinical onset to first seeing a psychiatrist, when this was the first specialist referral, was eight months (mean and median) with a range of two weeks to 16 months. In cases in which the initial referral was to a neurologist the interval to seeing a psychiatrist was four weeks median, 10 weeks mean, with a range of 2-38 weeks. All patients were investigated by a neurologist at some stage and the time from first psychiatric appointment to first neurological appointment was nine weeks mean, eight weeks median with a range of one day to 32 weeks.

\section{Psychiatric features}

The psychiatric symptoms in the first 14 cases of new variant $C J D$ have been described previously in detail (Zeidler et al, 1997b). The symptomatology in the subsequent 19 cases is very similar to the initial cases. The grouped data on 
Table 1. Psychiatric symptoms in 33 cases of new variant Creutzfeldt-Jakob disease

\begin{tabular}{lc}
\hline Pyychiatric symptom & Cases $(n)$ \\
\hline Depression & 16 \\
Anxiety & 17 \\
Withdrawal & 19 \\
Aggression/irritability & 18 \\
First-rank symptoms & 5 \\
Hallucinations & 17 \\
Delusions & 18 \\
Forgetfulness at onset & 4 \\
Suicidal ideation & 3 \\
\hline
\end{tabular}

the psychiatric symptoms in the total of 33 cases of new variant CJD are summarised in Table 1 .

All but one case exhibited psychiatric symptoms in the early stages of the illness and in most of the cases the psychiatric symptoms were the first manifestation of the illness. The single case without definite psychiatric symptoms was emotionally labile for some months prior to the onset of neurological symptoms and signs. The psychiatric symptoms initially occurred in isolation in the majority of cases, but in four there was a description of forgetfulness at the onset in addition to the psychiatric symptoms. In 17 cases pain in the limbs or joints, or painful or unpleasant paraesthesia or dysaesthesia was present, usually in combination with the psychiatric symptoms and prior to the development of other neurological symptoms and signs some months later. In six cases the painful limb or sensory symptoms were present from the onset and in all cases the pain or sensory symptoms persisted for weeks or months. Other early neurological symptoms during the 'psychiatric phase included gait imbalance and in a small minority of cases blurred or double vision, dysarthria, dysgeusia, involuntary movements or dysgraphia. A few patients stopped driving, some after road traffic accidents.

Seventeen patients suffered visual or auditory hallucinations and five cases exhibited first-rank symptoms. Aggression and irritability were prominent features in 18 cases and in a minority this was associated with tantrums or aggressive outbursts, including a small minority with violent behaviour. In two cases there was transient suicidal ideation and one patient wrote notes indicating suicidal intent.

Fleeting delusions were noted as an unusual feature in the original descriptions of the psychiatric features of new variant CJD. Of the subsequent 19 cases, eight described fleeting delusions, including beliefs that a child was missing, that a child had been killed, that the house had burnt down, that they were pregnant and that they were possessed by the devil. In contrast to the flitting delusions previously described, in two of the subsequent cases the delusions became persistent. The psychiatric diagnosis in the majority of cases was depression, although in some cases the possibility of an organic basis was suspected clinically, particularly in those cases with associated forgetfulness. In a small number of cases a psychotic illness was suspected and in two cases the psychiatric symptoms were thought to be hysterical or functional.

The psychiatric treatment given to patients reflected these diagnoses and 19 patients received antidepressants, often initially prescribed by their general practitioner, five received antipsychotic medication and one electroconvulsive therapy. Ten patients received no specific psychiatric treatment.

\section{Diagnosis}

In a minority of cases the possibility of an organic basis to the psychiatric symptoms was suspected at an early stage, particularly in the small minority of cases with early cognitive impairment in addition to the psychiatric symptoms. Many patients were thought to be suffering from a psychiatric rather than neurological disorder because of the occurrence of psychiatric symptoms indicative of depression or psychosis, without any clinical symptoms or signs to suggest an underlying neurological disorder.

All patients were referred for a neurological opinion when symptoms of neurological dysfunction developed, often months after the initial onset of psychiatric symptoms. Some patients were seen by neurologists and then referred to psychiatrists because the predominantly psychiatric symptomatology suggested that the neurological symptoms might have a psychiatric rather than a neurological basis. In a small number of cases there was a difference of opinion between the psychiatrist and the neurologist about the nature of the underlying disease process. Even when neurological symptoms developed, neurological signs were often minimal or absent.

One case of new variant CJD was examined by a consultant neurologist 15 months after the disease onset and there were no abnormal neurological signs despite prominent neurological symptoms.

Investigations during the psychiatric phase were almost always unhelpful. A minority of cases underwent electroencephalogram (EEG), computerised tomography or magnetic resonance imaging (MRI) brain scans prior to neurological referral and, with the exception of an EEG showing slow-wave activity, these investigations were reported to be normal. It is of note that the EEG in a small number of cases of new variant CJD was normal at a stage when there was clear evidence of neurological 
abnormalities on clinical examination and one MRI brain scan was normal during the early stages and became abnormal, with a posterior thalamic high signal, at a later stage when there were more prominent neurological signs.

The CJD Surveillance Unit has details of neuropsychological assessments carried out in 10 cases. All of these examinations showed clear evidence of cognitive impairment, but the majority of these examinations were carried out after the development of neurological signs and some after neurological assessment. Neuropsychological assessment was carried out at an earlier stage in two cases, two months and three months prior to neurological assessment and four months and two months respectively after the onset of symptoms. Both assessments demonstrated evidence of cognitive impairment, although one of these cases had forgetfulness as an initial symptom.

\section{Discussion}

The diagnosis of new variant $\mathrm{CJD}$, and its distinction from common psychiatric disorders, may not be possible in the early stages of the illness. Even after neurological symptoms develop there may be great difficulty distinguishing between psychiatrically determined neurological features and symptoms due to an underlying organic neurological disease. This problem is compounded by the transient improvement in the psychiatric symptoms in a small minority of these cases following treatment with antidepressants and in the individual who underwent electroconvulsive therapy. There is, however, an imperative to try and improve the early diagnosis of new variant CJD. The failure to make this diagnosis may later be interpreted by family members as a failure on the part of the psychiatrist and resentment that the patient was initially labelled as 'psychiatric' rather than suffering an organic illness. Delay in diagnosis is distressing to the relatives of patients, particularly in conditions such as new variant CJD in which there is a progressive decline in function requiring increasing levels of care.

There are some symptoms that may be helpful in suggesting the diagnosis of new variant CJD during the predominantly psychiatric phase, which may last for many months. The identification of cognitive impairment in addition to the psychiatric symptoms may raise the possibility of an underlying organic disorder in some cases. Persistent painful limb or joint pain, dysaesthesia or paraesthesia in combination with the psychiatric symptoms is unusual and may raise the suspicion of new variant CJD. Such symptoms include painful sensory disturbance in the hands and feet, in the face or mouth, or in discrete areas of the trunk. Some patients describe dysaesthesia in a similar distribution and some limb or joint pain. The persistence of these symptoms and their severity is distinct from the transient paraesthesia that may accompany anxiety and hyperventilation. Other suggestive neurological symptoms during the psychiatric phase include gait imbalance, visual disturbance, dysarthria, dysgeusia and involuntary movements, although it is understandable that these symptoms have in some cases been attributed to side-effects of medication.

Current evidence does not suggest that investigations such as EEG and MRI brain scanning are likely to provide useful diagnostic information before neurological signs develop. but few of these investigations were carried out in the early stages of the illness. Limited evidence suggests that neuropsychological assessment may demonstrate cognitive impairment at an early stage before neurological signs develop and such assessment should be considered if there is a clinical suspicion of organic basis to the psychiatric symptoms.

The descriptive evidence does, however, suggest that the diagnosis of new variant CJD may not be possible at an early stage in a significant proportion of cases as the clinical features are purely psychiatric. The difficulty in diagnosis is reflected in the referrals for a psychiatric opinion by neurologists even after there are neurological symptoms. The diagnosis of new variant $\mathrm{CJD}$ is difficult until neurological signs develop, particularly ataxia and involuntary movements such as myoclonus, chorea or dystonia. At this stage investigations such as an MRI brain scan are more likely to provide diagnostically useful information.

An important question is whether or not cases of new variant CJD would be identified earlier by the surveillance system if the psychiatric community were used as a source of case identification. The majority of cases of new variant CJD have been seen by psychiatrists, and in a small minority of cases the suspicion of the diagnosis of new variant CJD was raised by the psychiatrist. However, the median latency between the initial psychiatric appointment and obtaining a neurological opinion is only eight weeks and all patients with new variant CJD were referred for a neurological opinion. In occasional cases there was a prolonged period between psychiatric and neurological referral, but this may have reflected the gradual evolution of symptoms in some cases, rather than any delay in diagnosing an apparent organic disorder. The three cases with longest latency between psychiatric and neurological referral had durations of illness of 17 months, 23 months and 30 months in comparison with the median illness duration in new variant $\mathrm{CJD}$ of 14 months. Identification of cases of new variant 
CJD from psychiatric sources might allow the earlier referral of a minority of cases, but the evidence suggests that the psychiatric community are promptly referring cases of new variant CJD for a neurological opinion.

\section{Future occurrence and diagnosis}

New variant CJD is a rare disease, but there is a possibility that large numbers of cases could occur in future years. Psychiatrists should be aware of the psychiatric symptoms of this disease and of the diagnostic difficulties in the early stage. The diagnosis of new variant CJD is often not possible until the occurrence of frank neurological symptoms and signs, but the suspicion of this diagnosis may be raised in the early stages if there is evidence of cognitive impairment or persistent limb pain, paraesthesia or dysaesthesia in association with the psychiatric symptomatology. Other early neurological features in some cases include gait imbalance, dysarthria, blurring or double vision, involuntary movements, dysgeusia and dysgraphia.

Although there has been an extended latency in onward referral for a neurological opinion in a small number of cases, this probably reflects an extended, initially non-specific, psychiatric phase and overall there is only a short latency between psychiatric and neurological referral. Limited data suggests that there may be neuropsychological evidence of cognitive impairment prior to the development of neurological symp- toms. Early diagnosis is important to relatives of patients and the developments of early diagnostic markers is an important objective.

\section{References}

BRUCE, M. E., WILL, R. G., IRONSIDE, J. W., et al (1997) Transmissions to mice indicate that 'new variant' $\mathrm{CJD}$ is caused by the BSE agent. Nature, 389, 498-501.

WILL, R. G., IRONSIDE, J. W., ZEIDLER, M., et al (1996) A new variant of Creutzfeldt-Jakob disease in the UK. Lancet. 347, 921-925.

ZEIDLER, M. STEWART, G. E., BARRACLOUGH, C. R. et al (1997a) New variant Creutzfeldt-Jakob disease: neurological features and diagnostic tests. Lancet, 350, 903-907.

-. JohnStone. E. C., BAMBER, R. W. K., et al (1997b) New variant Creutzfeldt-Jakob disease: psychiatric features. Lancet, 350, 908-910.

*R. G. Will, Professor of Clinical Neurology, University of Edinburgh and Director of National CJD Surveillance Unit, Western General Hospital, Edinburgh EH4 2XU; G. Stewart, Research Registrar, National CJD Surveillance Unit; M. Zeidler, Research Registrar, National CJD Surveillance Unit; M. A. Macleod Research Registrar, National CJD Surveillance Unit and R. S. G. Knight, Consultant Neurologist, National CJD Surveillance Unit and Western General Hospital, Edinburgh

*Correspondence

\title{
Survey of suicides in the Fife region of Scotland
}

\author{
Fergus Douds and Vicky Bridges
}

\begin{abstract}
Aims and methods Suicides in the Fife region were investigated over a two-year period. The timing of the final contact with general practitioners and psychiatric services was ascertained. Data were collected from procurators fiscal records, general practices, and where applicable, psychiatric records.

Results There were 74 suicide victims during the study period. Forty-six per cent of suicide victims saw their general practitioner in the month before death, and $55 \%$ had a history of previous contact with psychiatric
\end{abstract}

services, although only $27 \%$ of this group saw a psychiatrist in the month before death.

Clinical implications Clinical audit of suicide is an important task for psychiatric senvices. Practitioners must continually assess risk and attempt, where possible, to reduce risk factors.

The Fife region, situated north of Edinburgh, has a mixed urban/rural population of 350000 